\title{
New Directions for the National Institutes of Health (NIH) Office of Behavioral and Social Sciences Research (OBSSR)
}

Robert M. Kaplan, Associate Director for the Behavioral and Social Sciences;

Director, Office of Behavioral and Social Sciences, NIH

7 he National Institutes of Health (NIH) is a complex organization with 27 Institutes and Centers (ICs). As one of four programmatic offices within the NIH

1 Office of the Director, the mission of the Office of Behavioral and Social Sciences Research (OBSSR) is to coordinate and stimulate behavioral and social science research across all NIH institutes and centers. Since OBSSR does not fund research directly, collaboration with the ICs is crucial. Although OBSSR is not in a position to control what individual IC directors may choose to fund or focus upon, behavioral and social science activity is common across the ICs, - both in terms of basic research on behavioral and social mechanisms affecting health, and translational research on converting knowledge into practice.

In addition to OBSSR's coordinating role, members of an NIH-wide behavioral and social science research coordinating committee meet monthly to keep one another informed and to streamline and leverage their various activities, as needed.

How much behavioral and social science research actually takes place at NIH? OBSSR is exploring this question more systematically and in some detail,
NIH Mission Statement NIH's mission is to seek fundamental knowledge about the nature and behavior of living systems and the application of that knowledge to enhance health, lengthen life, and reduce the burdens of illness and disability.
(NIMH) is the biggest player, followed by the National Institute of Drug Abuse (NIDA), the National Institute on Child Health and Development (NICHD), the National Institute on Aging (NIA), NIAAA, and the National Cancer Institute (NCI) (See Figure 1).

OBSSR's priorities need to be aligned and coordinated with those of NIH overall and the broader notions of health are embedded in NIH's mission statebut until that analysis is completed, a reasonable estimate might be around 1112 percent of the total NIH budget. Although all the ICs report at least some behavioral and social science activity, the National Institute on Mental Health ment. In particular, five opportunities for research at NIH that Dr. Collins presented in a Science article (Collins 2010) soon after he assumed the NIH Director role:

- High-throughput technologies; 
- Translational medicine;

- Benefitting health care reform;

- Focusing more on global health; and

- Reinvigorating and empowering the biomedical research community.

Some of the work is already underway at OBSSR and elsewhere within NIH. Specific opportunities include:

\section{Back to basics}

OBSSR is home to the NIH-wide OppNet program, which represents a significant and ongoing investment in strengthening the relationship between basic biomedical and behavioral/social science research across the agency.

Taking advantage of current and future innovation

While high-throughput technology has not been a major focus of activity (yet), gene-environment interaction (GEI) is - and promises to be even more so in the future. OBSSR is active in the GEI area by linking the exposure biology program (which is developing technologies and biomarkers for tracking diet, physical activity, environmental exposures, psychosocial stress and addictive substances) with research on genetic variants.

Current OBSSR and NCI-funded research being conducted by Kevin Patrick at UC San Diego on the "exposome" - a concept comparable to the human genome, but that measures instead the exposures (including lifestyle factors as well as environmental ones) that a human experiences across the life course and explores the relationships between these exposures and disease (Wild 2005). In many respects, measuring genotypes is fairly straightforward, compared to the dynamic, lifelong exposures that evolve continuously, starting in utero and continuing through childhood, adolescence, adulthood and old age. Gene and environmental exposure should be considered together, but currently there's an imbalance in the tools for measurement.

With the widespread adoption of mobile phone technology (currently at 5 billion users worldwide and expected to quadruple to 20 billion within the next decade) and applications for phones and other devices, changes in how health is portrayed, measured, and understood will follow.

Certainly, there is justifiable skepticism that some new apps will merely offer glitzier versions of "old school" (and ineffective) health education messages. Still, the technology is likely to change very rapidly, offering potential uses we can't even envision yet. For example, some basic cell phone apps exist that allow an individual to take an overhead picture of a plate of food at a meal, and estimate the intake of protein, carbohydrates, fat, etc. (Borrell 2011). The technology is crude at the moment, but won't be for long - new iterations are already in development. Similarly, sensors and nanotechnology are already becoming feasible means of capturing and relaying information in real time such as merging GPS and activity data to determine which park features support the most physical activity.

The point is that we have to think ahead, and try to imagine where the research will be in 15 or 20 years. "Will we have the right technologies?" "Are we thinking far enough ahead?" OBSSR is trying to think ahead in a number of 
ways, considering the implications for new research designs, new methods of data fusion and synthesis, new generations of health data analysts, and new approaches to handling the privacy of health-related information and health data security. Specific OBBSR activities and partnerships in these areas include a Systems Science Institute conference held in Pittsburg in May 2011, mHealth with Qualcomm in June 2011, a “Big Data" initiative on data visualization, and exploring ways to harmonize psychosocial information in EMRs.

\section{Informing public policy}

Benefitting health care reform was another item on Dr. Collins's "opportunities" list; he would like to see NIH more involved in presenting evidencebased opinion that could provide insights for public policy. This represents a new direction for NIH, which typically has shied away from public policy research. For OBSSR, which is a natural home for these activities, it has meant participating actively in a CMS working group and becoming more involved in partnerships with the Centers for Disease Control and Prevention (CDC).

One area of interest is the negative relationship between the quality of health care and Medicare spending. Iowa, for example, is on the low end of expenditures but the high end of quality, while California spends nearly the most yet achieves the very low quality in relation to other states. As an example, consider my two former hometowns in southern California, just 100 miles apart: Los Angeles and San Diego. The Medicare program spends an average of $\$ 11,300$ for each recipient in Los Angeles, in comparison to $\$ 8,500$ in San Diego
(Kaplan 2011). To illustrate how the importance $\$ 2,800$ difference in expenditures could be in two communities sharing such geographic proximity, consider the cost of a Lexus ES 300 - a luxury car that currently retails for just under $\$ 52,000$. The car's cost represents the difference in median Medicare costs between Los Angeles and San Diego counties (based on Dartmouth Atlas data) for a 65-year-old with a projected 18.6-year life expectancy from that point forward $(\$ 2800 \times 18.6$ years $=\$ 52,080)$. Simple calculations suggest that you could afford to buy a brand-new Lexus for every Medicare recipient in Los Angeles (1.3 million people) if they would accept the medical care one gets in San Diego instead. Getting more for less, and getting higher health care quality at lower costs, are questions to pursue and document.

\section{Answering the dissemination challenge}

Although we spend billions to create the next generation of therapies and cures, we spend a very small proportion of that investment - as little as \$.01 for every $\$ 1$ - on learning how to disseminate those same treatments (Woolf and Johnson 2005). For example, treatment of high blood pressure to control hypertension is an area we can take pride in.

According to NHANES data from 2004, awareness of hypertension among those with high blood pressure reached 80 percent (Egan, Zhao et al. 2010). But only 70 percent of these patients were in treatment, and only 30 percent had their blood pressure under control. The latest data show some improvement, with control as high as 50 percent. However, even in this scenario, if 80 percent are aware, 70 percent are under treatment, 
and 50 percent of patients have their high blood pressure under control, this "improved" level of control still means that only 28 percent of those with high blood pressure are successfully treated. We know from clinical studies that almost anyone can have his or her blood pressure controlled: It's a problem of dissemination.

Comparisons of investments in tweaking molecules versus dissemination show a far greater return on investment for investments in dissemination. For example, office reminders for cholesterol treatments were estimated to prevent seven times more deaths than replacing older cholesterol-lowering drugs with more potent versions of the drugs (Woolf and Johnson 2005).

To support dissemination and translation research, OBSSR is sponsoring conferences, training institutes, and trans-NIH Funding Opportunity Announcements (FOAs) that highlight different aspects of dissemination, translation, and implementation.

\section{Going global}

Global health issues are another opportunity on Dr. Collins's list. Although life expectancy for females has increased steadily in developing countries since 1960, life expectancy increases for women in the United States are not keeping pace with the rest of the world, with the United States now ranked $47^{\text {th }}$ (Crimmins, Preston et al. 2011). OBSSR is working with the National Academy of Sciences and Institute of Medicine on a project to explore international health differences in high-income countries and identify public health opportunities. The Office values global health, recognizing the importance of global health in an in- creasingly interconnected world. More specifically, behavioral and social science research clearly has a significant role to play in reducing the global burden of disease. OBSSR is active in several workforce and research training initiatives in Africa, most recently convening a week-long institute in South Africa that brought together 45 researchers from 13 sub-Saharan countries.

While OBSSR has been active in each of these areas and will continue to be, we also want to think about what the world - and science - will look like in the next decade or two, and how we can best prepare for it. Discussions with leaders of academic research institutions and with faculty research innovators will help guide future NIH policy and research agendas.

\section{References}

Borrell, B. (2011). "Epidemiology: Every bite you take." Nature 470(7334): 320-322.

Collins, F. S. (2010). "Research agenda. Opportunities for research and NIH." Science 327(5961): 36-37.

Crimmins, E., S. Preston, et al., Eds. (2011). International Differences in Mortality at Older Ages: Dimensions and Sources. Washington, National Academy Press.

Egan, B. M., Y. Zhao, et al. (2010). "US trends in prevalence, awareness, treatment, and control of hypertension, 1988-2008." JAMA 303(20): 2043-2050.

Kaplan, R. M. (2011). "Variation between end-oflife health care costs in Los Angeles and San Diego: why are they so different?" J Palliat Med 14(2): 215-220.

Wild, C. P. (2005). "Complementing the genome with an "exposome": the outstanding challenge of environmental exposure measurement in molecular epidemiology." Cancer Epidemiol Biomarkers Prev 14(8): 1847-1850.

Woolf, S. H. and R. E. Johnson (2005). "The breakeven point: when medical advances are less important than improving the fidelity with which they are delivered." Ann Fam Med 3(6): 545-552. 
NINR, NCMHD, NEI, NIEHS,

RMAP, OD, NIGMS, NCRR,

NIAMS, NCCAM, NIDCR,

FIC, NHGRI, NLM, NIBIB

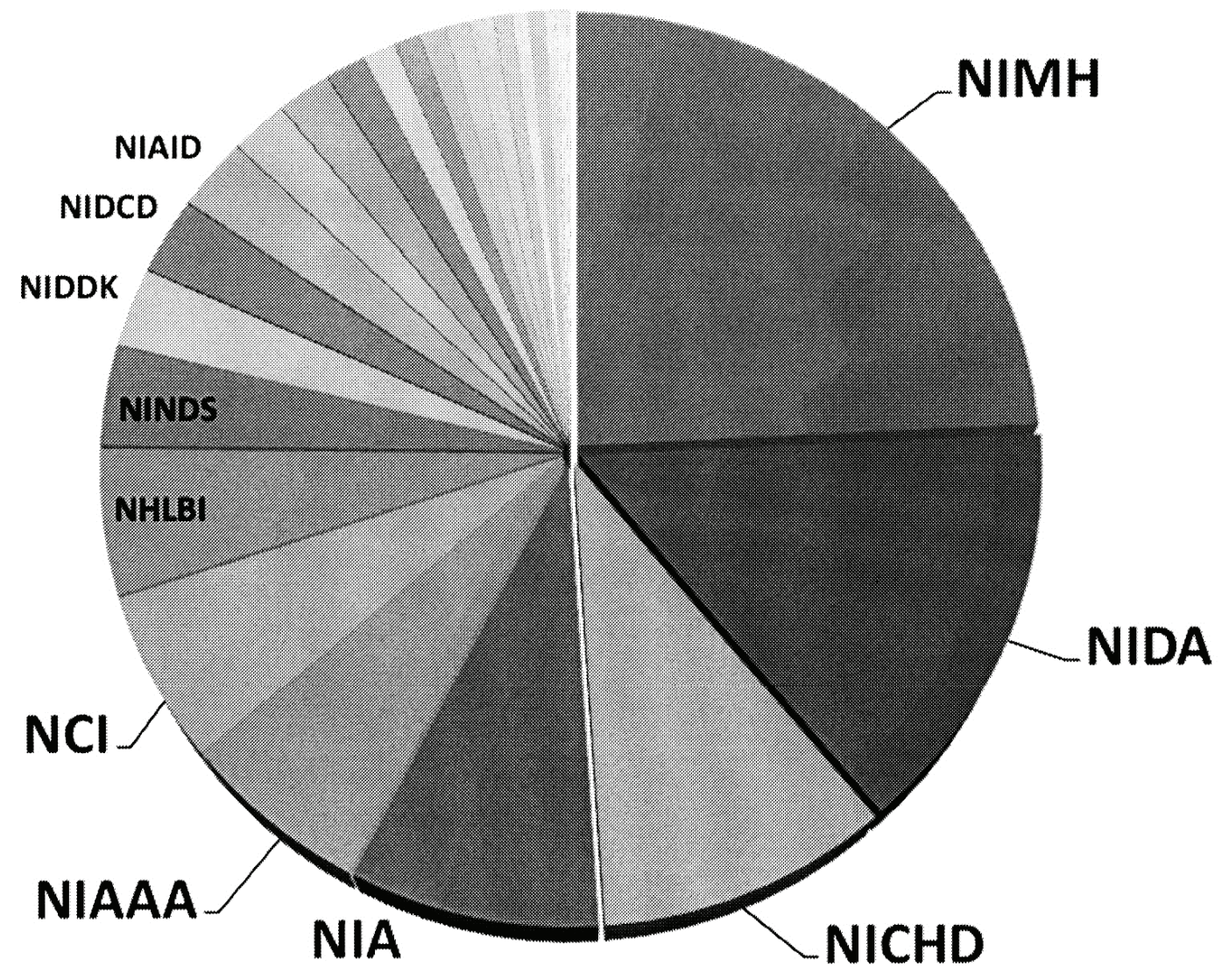

Figure 1. Fiscal Year 2010 NIH Behavioral and Social Science Research Funding by Institute of Center. Total non-ARRA funding was $\$ 3.53$ billion 